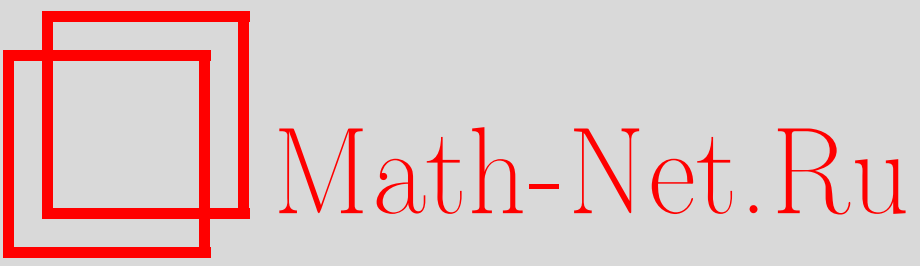

А. Эршлер (Дюбина), Асимптотики сноса и энтропии для случайного блуждания на группах, УМH, 2001, том 56, выпуск 3, 179-180

DOI: https://doi.org/10.4213/rm411

Использование Общероссийского математического портала Math-Net.Ru подразумевает, что вы прочитали и согласны с пользовательским соглашением

http://www.mathnet.ru/rus/agreement

Параметры загрузки:

IP : 52.23 .180 .231

26 апреля 2023 г., 09:34:36 


\title{
АСИМПТОТИКИ СНОСА И ЭНТРОПИИ ДЛЯ СЛУЧАЙНОГО БЛУЖДАНИЯ НА ГРУППАХ
}

\author{
А. ЭРшлЕР (ДюБИнА)
}

Мы рассматриваем симметричные случайные блуждания на группах такие, что носитель соответствующей меры $\mu$ конечен и порождает группу. Каждому такому случайному блужданию мы можем сопоставить функции

$$
H(n)=-\sum_{g \in G}\left(\mu^{* n}\right) \ln \left(\mu^{* n}(g)\right) \text { и } L(n)=\mathrm{E}_{\mu^{* n}} l(g),
$$

где $l$ - словарная метрика, а $\mu^{* n}-n$-я свертка меры $\mu$. Функция $H(n)$ называется әнтропией, а $L(n)$ - сносом (или скоростью ухода на бесконечность) случайного блуждания. $H(n)$ измеряет, насколько равномерно распределена мера $\mu^{* n} . L(n)$ показывает, насколько быстро случайное блуждание удаляется от своей начальной точки. Известно, что $H(n)$ асимптотически линейна тогда и только тогда, когда $L(n)$ асимптотически линейна [1], и тогда и только тогда, когда граница Пуассона нетривиальна [2]. В частности, это так для любой неаменабелшной группы. С другой стороны, во многих других примерах $L(n)$ асимптотически ведет себя как $\sqrt{n}$.

Первые примеры нестандартного роста $L(n)$ были построены автором в [3], [4]. В этих приме$\operatorname{pax} L(n) \asymp n^{1-\frac{1}{2^{k}}}(k-$ натуральное) и $L(n) \asymp n / \ln (n)$.

В этой статье мы анонсируем новые примеры промежуточного роста сноса и оценки для промежуточных асимптотик энтропии.

ОпредЕлЕниЕ. Напомним, что сплетением групп $A$ и $B$ называется полупрямое произведение $A$ и $\sum_{A} B$, где $A$ действует сдвигом на $\sum_{A} B$ : если $a \in A, f: A \rightarrow B, f \in \sum_{A} B$, то $f^{a}(x)=f\left(x a^{-1}\right), x \in A$. Обозначим сплетение $A$ 々 $B$.

Теорема 1. 1. Пусть F-конечная группа. Рассмотрим следующие группь, определенные рекуррентно:

$$
G_{1}=\mathbb{Z}^{2} \imath F ; \quad G_{i+1}=\mathbb{Z}^{2} \imath G_{i} .
$$

Тогда для некоторого простого случайного блуждания на $G_{i}$ и для любого достаточно большого $n$

$$
L_{n}^{G_{i}} \asymp \underbrace{\frac{n}{\ln (\ln (\ldots \ln (n) \ldots))} .}_{i}
$$

2. Рассмотрим следующие группь, определеннье рекуррентно:

$$
F_{1}=\mathbb{Z} ; \quad F_{i+1}=\mathbb{Z} \imath F_{i}
$$

u nycmb

$$
H_{1, i}=\mathbb{Z}^{2} \imath F_{i} ; \quad H_{j+1, i}=\mathbb{Z}^{2} \imath H_{j, i} .
$$

Тогда для некоторого простого случайного блуждания на $H_{j, i}$ и для любого достаточно большого $n$

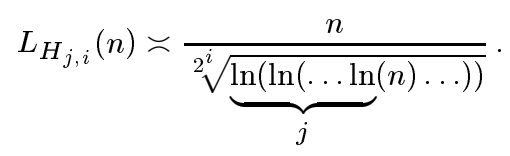

Основным техническим средством доказательства является следующее утверждение. В нем мы рассматриваем простое случайное блуждание на $\mathbb{Z}^{2}$. Пусть $b_{z}^{(n)}-$ это количество раз, которое случайное блуждание посетило элемент $z\left(z \in \mathbb{Z}^{2}\right)$ до момента $i$. 
УТВеРЖДЕНИЕ. Пусть $T>0, a f-$ вогнутая возрастающая функция такая, что

1) для любого $C>1 C f(x) \geqslant f(C x)$,

2) $f(0)=0$ и существует $x$ такое, что $f(x)>1$,

3) для любого $n \geqslant 1$ функция $x f(n / x)$ вогнута на отрезке $(0, n / T]$.

Тогда для достаточно больших $n$

$$
\mathrm{E}\left(\sum_{z \in \mathbb{Z}^{2}} f\left(b_{z}^{(n)}\right)\right) \asymp f(\ln (n)) \frac{n}{\ln (n)}
$$

Оценим теперь энтропию случайного блуждания на группе. Известно (см. [5]), что для широкого класса мер на нильпотентных группах $H(n) \asymp \ln (n)$. Как уже отмечалось, для любой неаменабельной группы энтропия $H(n)$ асимптотически линейна. Мы покажем, что существует бесконечно много промежуточных асимптотик роста энтропии.

Пусть $v(n)$ - количество элементов группы длины не болшше $n$,

$$
v=\lim _{n \rightarrow \infty} \frac{\ln (v(n))}{n} .
$$

Следующая лемма обобщает фундаментальное неравенство из [6], [7].

Лемма 1. Для любого $\varepsilon>0$ существует $C>0$ maкое, что

$$
H(n) \leqslant(v+\varepsilon) L(n)+\ln (n)+C .
$$

Другая лемма оценивает энтропию снизу. Эта лемма является следствием резултата из [1].

Лемма 2. Существует $C>0$ maкое, что

$$
H(n) \geqslant C \mathrm{E}_{\mu * n} l^{2}(g) / n-\ln (n) \geqslant C L^{2}(n) / n-\ln (n) .
$$

Как следствие из двух предыдущих лемм и теоремы 1 мы получаем следующую теорему.

Теорема 2. Пусть $G_{i}$ - это группа, определенная в теореме 1. Тогда для некоторого случайного блуждания на $G_{i}$

$$
\left.K_{1} n /(\underbrace{\ln (\ln \ldots \ln }_{i}(n) \ldots)\right)^{2} \leqslant H_{G_{i}}(n) \leqslant K_{2} n / \underbrace{\ln (\ln \ldots \ln }_{i}(n) \ldots)
$$

для некоторых положительных констант $K_{1}$ и $K_{2}$. Вчастности, все $G_{i}$ имеют разнье асимптотики әнтропии.

Автор выражает благодарность А. М. Вершику за постановку задачи и полезные обсуждения.

\section{СПИСОК ЛИТЕРАТУРЫ}

[1] N. Varopoulos // Bull. Sci. Math. 1985. V. 109. P. 225-252. [2] V. A. Kaimanovich, A. M. Vershik // Ann. Probab. 1983. V. 11. №3. P. 457-490. [3] А. Дюбина // УМН. 1999. Т. 54. № 5. С. 159-160. [4] А. Эршлер (Дюбина). Об асимптотике скорости ухода на бесконечность // Записки научн. семин. ПОМИ (в печати). [5] Y. Derriennic // Probability measures on groups VIII. Proc. 8th Conf., Oberwolfach/Germany, 1985. Berlin: Springer-Verlag, 1986. P. 241-284. (Lecture Notes in Math. V. 1210.) [6] A. M. Вершик // Записки научн. семин. ПОМИ. 1999. Т. 256. С. 1-11. [7] А. М. Вершик // УМН. 2000. Т. 55. № 4. С. 59-128. 